\title{
Premixed Combustion in a Periodic Flow Field. Part I: Experimental Investigation
}

\author{
J. O. KELLER* and P. K. BARR \\ R. S. GEMMEN \\ University of Michigan, Ann Arbor, MI 48109 USA
}

Combustion Research Facility, Sandia National Laboratories, Livermore, CA 94551-0969 USA

\begin{abstract}
An experimental investigation into the detailed mixing, combustion and ignition processes occurring in a flow field under the influence of resonant acoustic perturbations has been performed. These acoustic perturbations were created by a pulse combustor of the "Helmholtz" type. Cycle-resolved ensemble-averaged measurements of the velocity field, combined with instantaneous images of chemiluminescence and of schlieren, are presented. During the first two-thirds of the resonant cycle the inlet jet forms a well-defined toroidal vortex that is phase-locked with the combustor cycle. This toroidal vortex is responsible for the convection and mixing of the reactants with the residual hot products. Although chemiluminescence is always present in quiescent regions of the combustor, none exists in the region of the reactants during injection. Moreover, the combustion of the fresh reactants begins late in the cycle along the outer edges of the rolled up toroidal vortex, and not in the quiescent regions of the flow. The combustion of the reactants moves into the center of this toroidal vortex where rapid uniform ignition and subsequent combustion of the fresh reactants occurs. This flow field is dominated by a strong toroidal vortex which entrains on a large scale and mixes on a fine scale the fresh reactants with the residual hot products, preparing them for a rapid, almost volumetric, combustion process initiated by thermal and/or radical ignition.
\end{abstract}

\section{INTRODUCTION}

Acoustic interactions with combustion processes are the source of combustion instabilities in many systems (e.g., ramjets and rocket engines), and are the source of stable oscillations in devices such as pulse combustors. In systems such as ramjets these acoustic interactions can cause catastrophic failure, and in systems such as pulse combustors these interactions are desirable in that they cause significant increases in the rates of heat and mass transfer. Contemporary efforts in this area range from the study of acoustic interactions leading to flashback [1], the active control of instabilities by acoustic feedback [2], the study of acoustic fluctuations in ramjet-type combustors [3], the study of acoustic interactions in a low-speed dump combustor for potential use as hazardous waste incinerators $[4,5]$, and the study acoustic interactions in pulse combustors

\footnotetext{
${ }^{*}$ Corresponding author.
}

[6-8]. A good discussion of the acoustic interactions on the combustion process focusing on the chemical-acoustic interactions is presented by Oran and Gardner [9]. All of these problems are similar in that they all have combustion chambers with like geometries (sudden expansions, stagnation plate flame holders, etc.) and the fluid dynamics in the combustion zone and the combustion of the reactants are affected by the presence of acoustic vibrations. These vibrations may be in resonance depending on the interactions between the combustion process, the acoustics and the system geometry. Whether these interactions are viewed as undesirable (ramjets or rocket engines) or desirable (pulse combustors or some dump combustors) the acoustic interactions with the flow field and the subsequent combustion process are the same. The flow field in a pulse combustor is one in acoustic resonance and is very repeatable. Hence, the use of pulsating combustion is a convenient method to study the effects of resonant pressure fields on fluid dynamics and combustion processes. 
In the 1986 review article by Putnam et al. [10], a brief discussion of the fundamental operation of a pulse combustor is presented. They discuss a model of pulse combustor operation involving injection, ignition, and wave propagation. Although these authors pointed out that the assumptions used in their ignition model were questionable, their model represented the state-of-the-art at that time. Being aware of this issue investigations were initiated by two separate investigators to answer some of these questions [11-13]. Relying on spatially integrated data, these later works found that the measured chemiluminescence never went to zero, and thus concluded that combustion never ceased. Based on these results the combustion process has been described as one of flame propagation growing and shrinking in size during each cycle [14] or that the new reactants are ignited by remnants of reacting pockets from the previous cycle [13]. Much information is lost by the spatially integrated data presented in Refs. 11-13; it is distinctly possible that this continuous combustion source is not a discrete flame front. In support of this last argument, work by N. Ohiwa et al. [15] deduced from flame ionization measurements that combustion did not occur during the period of injection and in the region of the injected reactants. Based on these ionization measurements and schlieren images these authors concluded that a pair of large-scale eddy motions promoted mixing of hot products with the cold reactants, resulting in a distributed reaction zone.

More recently, the combustion process has been modeled as one in which the reactants first are mixed with residual products and then undergo thermal ignition [6,7]. Although this combustion model was successfully used by Barr et al. [16], these authors recognize that its validity relied on the success of the application. A more detailed understanding of the combustion and/or reignition process in a pulse combustor combustion chamber is needed.

Cycle-resolved measurements of the ensemble-averaged velocity field, combined with cycle-resolved instantaneous images of both chemiluminescence and schlieren photographs, are presented in this article. These data provide insight into the cycle-resolved velocity field and how it controls and is affected by the mixing and subsequent combustion of the reactants in the combustion chamber. This investigation is limited to steady state resonant conditions, (i.e., after the acoustic combustion instability has reached its limit cycle).

\section{FACILITY AND DIAGNOSTICS}

\section{Combustor}

Figure 1 presents a schematic of the facility used in this study. A one-way valve (flappervalve) is located in the reactant supply line just upstream of the square combustion chamber ( $80 \mathrm{~mm}$ square by $100 \mathrm{~mm}$ long). This valve opens and closes depending on the flow direction of the reactants from the supply line into the combustion chamber. For this work, the combustor was operated in a premixed mode where the fuel ( $99.99 \%$ methane) and air were mixed in a counterflow configuration upstream of the flapper valve. The injection system was configured so that the reactants were injected axially into the combustion chamber (with an inlet pipe diameter of $20.7 \mathrm{~mm}$ ), creating an axisymmetric velocity and temperature field similar to the one used in Ref. 17 . The reactants impinge on a stagnation plate, located on the centerline at a streamwise position of $x=$ $11.80 \mathrm{~mm}$ and with a diameter of $19.1 \mathrm{~mm}$. The square cross-section combustion chamber was connected to a square contraction section that joins the combustion chamber to the variable length tail pipe. For this work a tail pipe length

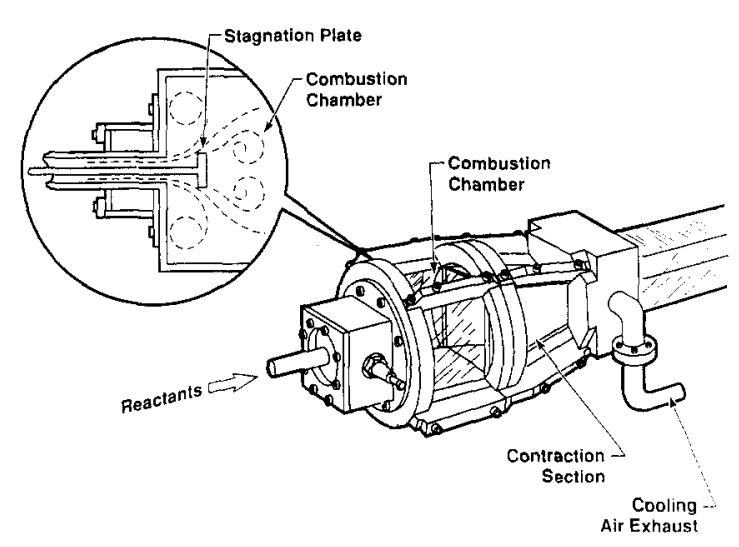

Fig. 1. Schematic of the valved pulse combustor showing the injection geometry. 


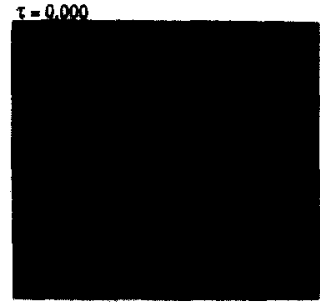

$\tau=0.055$

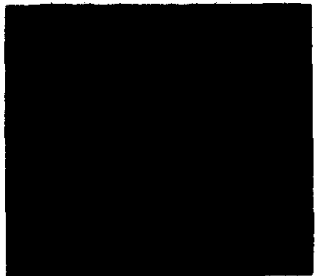

$\tau=0.111$

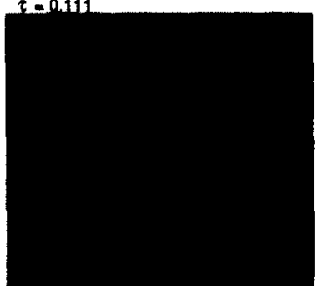

a)

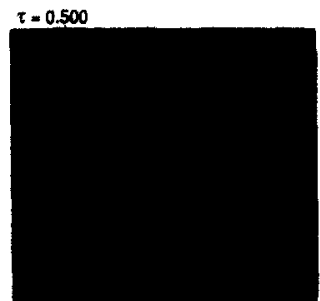

$t=0.558$

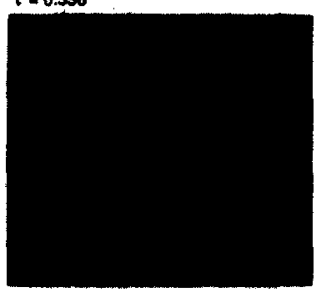

$\tau=0.611$

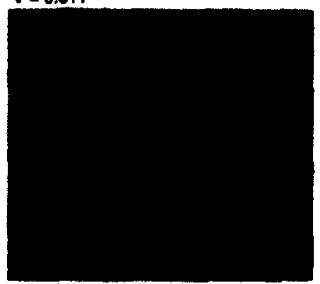

b)

Fig. 3. Cycle-resolved Schlieren images of the combusting flow in the combustion chamber. These images were taken with an exposure time of $20 \mu \mathrm{s}$ and were phaselocked with the combustor cycle, providing images for the first half (a) and the second half (b) of the cycle.
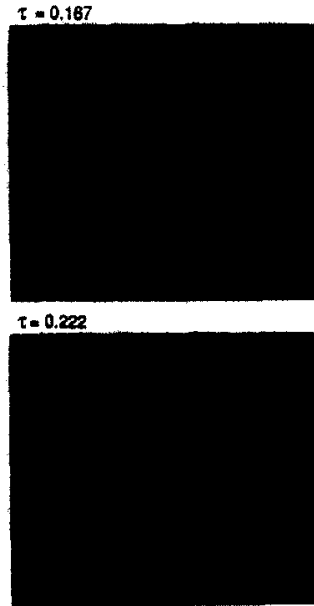

$t=0.278$
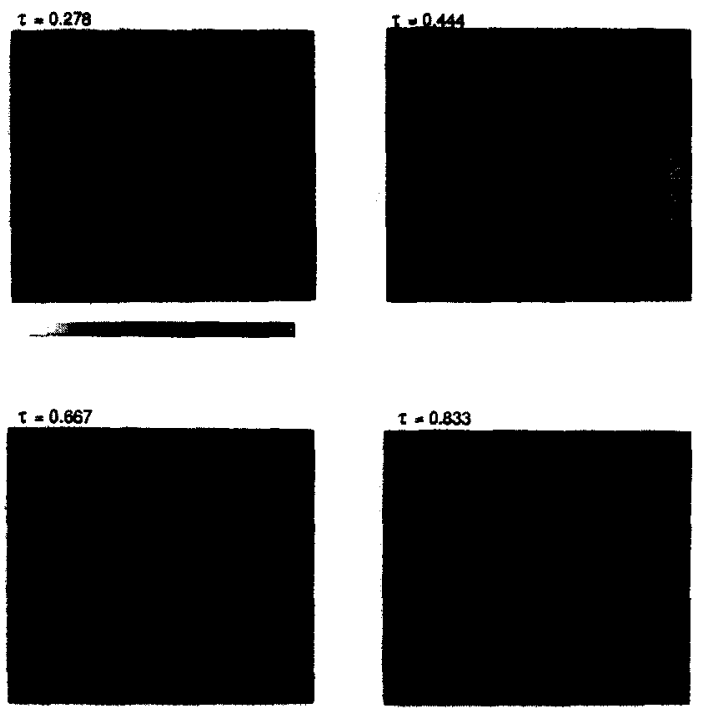

$t=0.722$

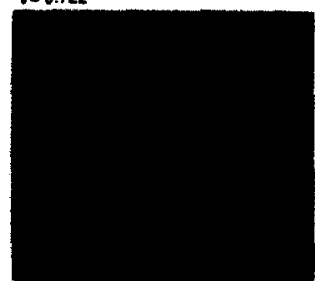

$I=0.778$

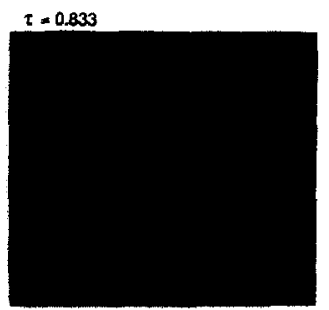

$t-0.39$

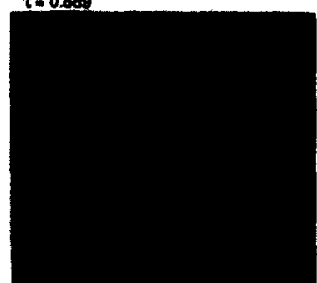

$\tau=0.994$
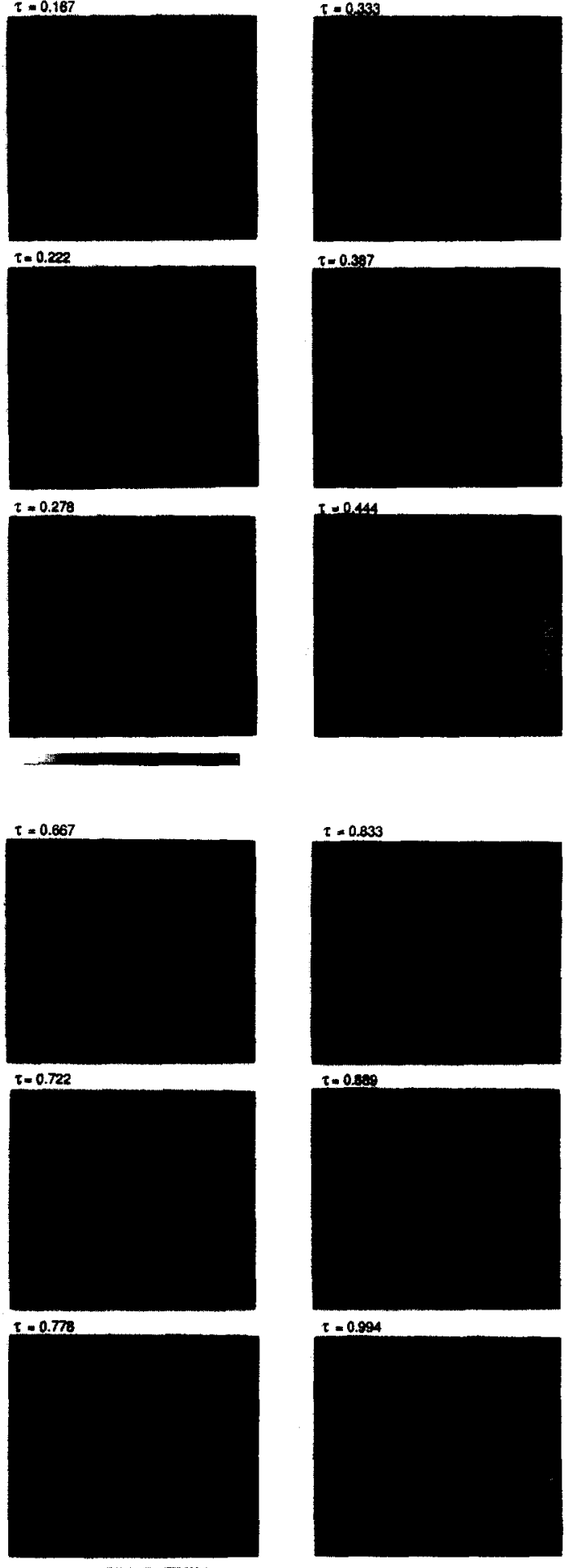

$\tau-0.287$
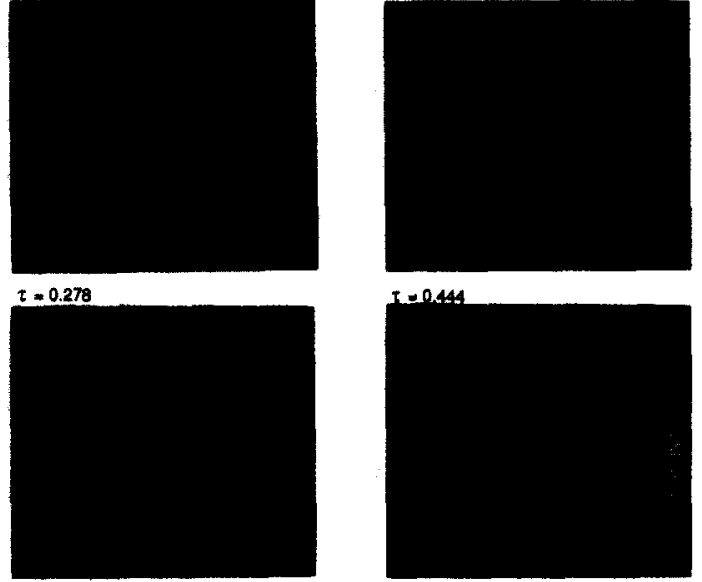

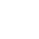


of $880 \mathrm{~mm}$ was chosen. At these conditions the combustor frequency is nominally $100 \mathrm{~Hz}$.

\section{Diagnostics and Operating Conditions}

Three different diagnostics were used here. Two components of gas velocity (streamwise, $U$, and transverse, $V$ ) were measured by laser doppler velocimetry (LDV). Laser schlieren photography was used to image the gradient of the refractive index caused by the interface between cold reactants and hot products, as well as by combustion. The laser schlieren system used here was of a standard configuration using square stop and a Bragg cell beam dump combination providing a high-speed shutter. Chemiluminescence was measured, providing both spatially resolved images in the $x-y$ plane (integrated along the line-of-sight) and volumetrically integrated information on the temporal location of reaction.

All measurements were cycle-resolved by phase-locking on the combustion chamber pressure. The downward-going zero-crossing of the oscillating component of pressure has been arbitrarily chosen as time zero. The phaselocked data for this work are presented in terms of the normalized cycle time $\tau$ which is defined to be $t / t_{\text {cycle }}$, where $t$ is the time in a given cycle and $t_{\text {cycle }}$ represents the total cycle time. For this work the combustor was operated at total mean mass flow rate of $4.54 \mathrm{~g} / \mathrm{s}$. For all the measurements of the combusting pulsating case, except for the schlieren photography, the equivalence ratio was set lean at $\phi=0.68$, yielding a nominal power input of 8.6 $\mathrm{kW}$. The combustor was allowed to reach thermal equilibrium by operating for $0.5 \mathrm{~h}$ before beginning the data acquisition.

To maximize the sensitivity of the schlieren system, these images were taken while the combustor walls were at ambient temperature. The measurements were performed immediately after starting the combustor, very early during the warmup transient to ensure the coldest possible wall conditions. Under these conditions the equivalence ratio had to be increased slightly to assure stable operation $(\phi$ $=0.75$ ). Obtaining the schlieren images while the combustor remained at ambient conditions and increasing the equivalence ratio increases the schlieren system's sensitivity. The temperature of the residual products from the previous cycle is increased, hence making the temperature gradient between the cold reactants and the hot products greater. Increasing the equivalence ratio will have an effect on the operating condition of the combustor [ 6 and 7], however, the major features of this flow field remain unaffected and correlate well with the other data presented in this work, since the data presented here are all normalized by the cycle time. Any change in the natural resonant frequency due to a change in the product temperature is accounted for in this normalization process.

The velocity was measured by a two-color, two-component LDV system. These data include both ensemble-averaged mean and ensemble-averaged fluctuating components. The mean data are presented in the form of velocity vectors, providing a mapping of the mean velocity field throughout the combustion chamber, whereas the fluctuating components are presented as intensity maps as a function of space and time. Velocity measurements were made to within $5-7 \mathrm{~mm}$ of the wall, a limitation caused by the transverse laser beam orientation. Unfortunately, $5-7 \mathrm{~mm}$ is well outside the momentum boundary layer, eliminating all of the near-wall information. Averages were performed over a sufficiently large sample size so that the data in the mean was statistically stationary. Ensemble-averaged velocity measurements were obtained by recording the time-of-arrival of each velocity realization and sorting the data into one of 18 time bins, based on the time in the cycle. Each time bin is approximately $660 \mu \mathrm{s}$ wide. Since the particle flux varied across the cycle, the number of realizations in each time bin varied from a minimum of 500 to a maximum of $35000{ }^{1}$ From these ensemble-averaged $\langle U\rangle$ and $\langle V\rangle$ velocity measurements, mean velocity vectors and velocity fluctuations were calculated as a function of time and space.

All cycle-resolved velocity data presented here have been corrected for known systematic

\footnotetext{
'See Refs. 8, 17, and 18 for more discussion on the ensemble averaging technique used here.
} 
errors, particularly those due to gradients in time and space (known as gradient broadening) [8]. However, no corrections for random errors, such as cycle-to-cycle variations, have been made. These errors will broaden the fluctuating components of velocity. Due to the data acquisition technique, any cycle-to-cycle variation in frequency shows up as a discontinuity in the fluctuating velocity component between the beginning and the end of the cycle. The cycleto-cycle variation in frequency is small, as indicated by the fact that these data correlate well at the beginning and end of the cycle.

Researchers have relied on chemiluminescence records to indicate qualitatively when and where combustion occurs in pulse combustors [see Refs. 6, 7, 13, and 19]. Two types of chemiluminescence records were obtained, total chemiluminescence from all sources (primarily $\mathrm{CC}^{*}, \mathrm{CH}^{*}$, and $\left.\mathrm{OH}^{*}[20,21]\right)$ and the chemiluminescence from one radical $\mathrm{OH}^{*}$. The choice of radical species to use for this type of qualitative measurement is somewhat arbitrary. Child [21] points out that chemiluminescence from $\mathrm{CH}^{*}, \mathrm{CC}^{*}$, and $\mathrm{OH}^{*}$ in methane air flames all occur in the reaction zone. The work of Keller and Westbrook [19] showed that the rate of production of $\mathrm{OH}^{*}$ peaks in conjunction with the peak in energy release. In addition, the time scale for $\mathrm{OH}^{*}$ decay to the ground state is approximately $1 \mu \mathrm{s}$ [20]. This makes $\mathrm{OH}^{*}$ a good candidate for the qualitative indicator of when and where energy release is occurring in this oscillating flow field. However, for the two-dimensional imaging of the chemiluminescence signal, at the desired short exposure times (less than $20 \mu \mathrm{s}$ ) the signal-to-noise ratio from $\mathrm{OH}^{*}$ was too low to provide a high-quality image. Therefore, light from all sources of chemiluminescence was obtained for the time-resolved images shown here. A comparison between these two chemiluminescence measurements (total emission and $\mathrm{OH}^{*}$ ) is presented in Fig. 2. These measurements were obtained by placing a photomultiplier tube (PMT) behind a pin hole located at the focal point of a lens positioned to collect parallel light emitted from the combustion chamber. The $\mathrm{OH}^{*}$ signal was obtained by placing a narrow band pass filter at $310 \pm$ $1.5 \mathrm{~nm}$ in front of the PMT. The data shown in

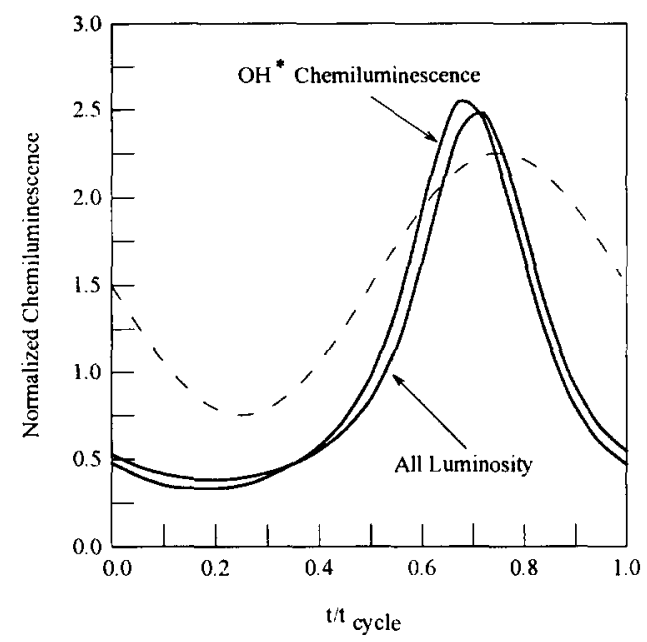

Fig. 2, Spatially integrated chemiluminescence measurements of the combusting flow in the combustion chamber. Shown is a comparison between $\mathrm{OH}^{*}$ and all luminosity produced by the combustion of $\mathrm{CH}_{4}$. The dashed line represents a typical combustion chamber pressure shown for phase relationship information only.

Fig. 2 are normalized so that the area under each curve is unity and are presented as a function of normalized cycle time. The general shape of these two curves is the same with only a minor difference in the temporal location of the peaks. As shown in Fig. 2, the peak in the $\mathrm{OH}^{*}$ chemiluminescence occurs before the peak in the spectrally integrated chemiluminescence. This difference is approximately 400 $\mu \mathrm{s}$, which is $2 / 3$ of the time between our images. The dashed curve is a typical combustion chamber pressure profile included here to show that the majority of the energy is released during the high-pressure portion of the cycle.

\section{DATA PRESENTATION}

\section{Schlieren Images}

Care was taken in the design of this combustion chamber to provide an axisymmetric flow pattern while maintaining good pulsating conditions. As sketched in Fig. 1, the location of the stagnation plate creates a conical jet. This injection profile is quite evident in the cycle-resolved schlieren images presented in Figs. 3a and $3 \mathrm{~b}$ (See color plate between pages 30 and 31.). These data are organized in two plates, each with 9 images or plots. The color scale is 
indicated at the bottom of each of these figures, with the intensity of the signal increasing to the right.

Figure $3 \mathrm{a}$ presents data during the first half of the cycle (negative gage pressure), and Fig. $3 \mathrm{~b}$ presents data during the second half of the cycle (positive gage pressure). The injection of the cold reactants during the first $67 \%$ of the cycle is shown in these schlieren images. The reactants are injected into the residual hot products, which show little inhomogeneity in the gradient of the refractive index, implying that this fluid is reasonably well mixed and isothermal. The interface that is depicted during the injection process could be due to either inhomogeneity caused by cold reactants and hot products or energy release due to combustion. As the cycle continues the injection of reactants penetrates further into the combustion chamber $(\tau=0.333)$, these images show the existence of a structure as far downstream as $x=50$ to $70 \mathrm{~mm}$ (see $\tau=0.444$ ). This could be due to either the penetration of cold reactants, or the release of energy, or both. Recall from Fig. 2 that the chemiluminescence signal remains low for the first half of the cycle, then starts to increase rapidly to a peak at $\tau \simeq 0.7$, and then decreases rapidly to near its mininum value at $\tau=1.0$. The presence of unmixed reactants and products is evident during the onset of combustion ( $\tau \simeq 0.4$ ), but is seen to be less conical and is pushed back against the front of the combustion chamber. Note that the region near the injector never becomes homogeneous.

\section{Chemiluminescence Images}

Shown in Figs. $4 \mathrm{a}$ and $4 \mathrm{~b}$ (See color plates between pages 30 and 31.) are instantaneous images of the total chemiluminescence emitted in the combustion chamber. The combustion chamber was imaged onto the camera's detector by a small $f$ number $(f \simeq 1.0$ ) lens system, causing a reasonable degree of parallax. The green outline shown in these figures depicts the edges of the combustion chamber walls as well as the injection port and the stagnation plate. The background signal has been removed from these data (shown in gray). The chemiluminescence at $\tau \simeq 0.222$ corresponds to the minimum chemiluminescence shown in Fig. 2. The color scale for these images is also located at the bottom of each image. Unlike the schlieren images of Fig. 3, the increase in intensity was chosen to progress from dark blue to white, indicating an increase in the chemiluminescence intensity.

Investigation of the chemiluminescence images at times near $\tau=0$ show a small but distinct signal of chemiluminescence in the upper part of the combustion chamber, near the entrance (shown as a dark blue). With increasing cycle time the signal decreases to a minimum at $\tau \simeq 0.222$. Close examination of the images at times $0.278 \leq \tau \leq 0.444$ shows a small but gradual increase in intensity at the regions behind the stagnation plate and far downstream into the combustion chamber. This becomes particularly evident at $\tau=0.444$, distinctly showing reaction occurring in a " $V$ " like structure originating at the downstream side of the stagnation plate, expanding and extending the entire length of the combustion chamber (note the small magenta spot far downstream). This intensity continues to increase through a peak at $\tau=0.7$ in agreement with the data of Fig. 2. The intensity rapidly decreases as the reactants are consumed, leaving residual combustion to occur in the quiescent regions near the top wall and behind the stagnation plate ( $\tau=0.0$ and $\tau=0.055$ ).

These data combined with the schlieren images of Fig. 3 help to clarify the schlieren images shown in Fig. $3 b$ describing the rapid combustion period (times $0.4 \leq \tau \leq 0.9$ ). Since the spatial location of the schlieren image is near the combustion chamber entrance and not downstream where combustion is occurring, the schlieren images shown during the period of rapid combustion are due to the interface between cold reactants and hot products and not combustion of these reactants.

\section{Velocity Field}

The velocity field along the center plane for the entire $x-y$ field under a noncombusting, nonpulsating condition is shown in Fig. 5. This flow field is dominated by a large recirculation zone with positive streamwise velocity along the outer edges and negative streamwise veloc- 


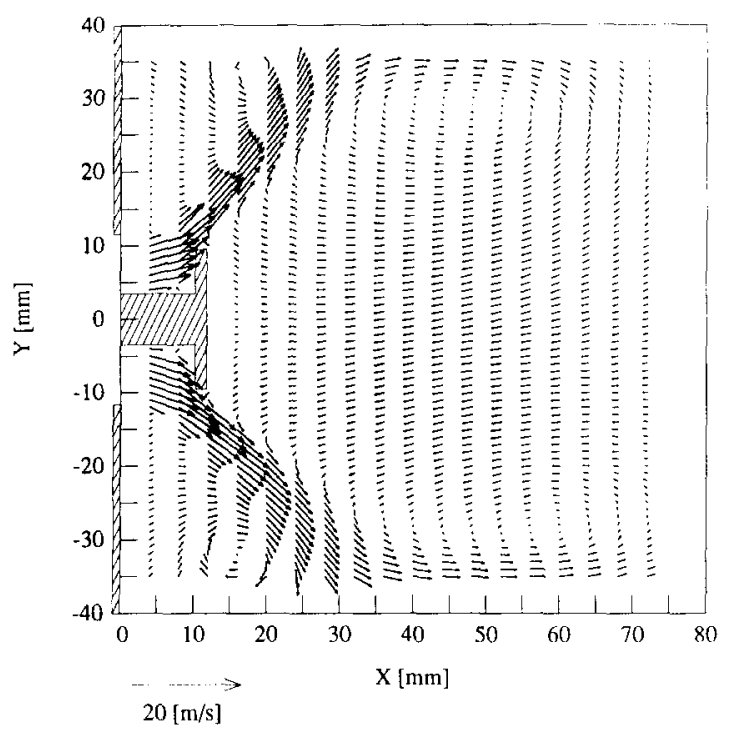

Fig. 5. Velocity field mapping in the combustion chamber for noncombusting nonpulsating flow.

ity along the centerline. Close examination of the outside portion of the inlet jet shows the existence of a smaller yet well-defined recirculation eddy $(x=15 \mathrm{~mm}$ and $y=-32 \mathrm{~mm})$. From the data presented in Fig. 5 it is evident that a significant portion of the flow moves along the wall (outside of the measurement domain).

Cycle-resolved ensemble-averaged velocity data were obtained for the reacting pulsating case and are presented in Fig. 6a and $6 \mathrm{~b}$. These data are organized in the same fashion as are Figs. 3 and 4 . Due to the quantity of data and the level of difficulty in acquisition for the pulsating case, the domain over which data was acquired was restricted to the lower half of the combustor. For the purposes of this work symmetry in the flow field was used to create the LDV data shown at the top half of the combustor.

The dominance of the injection of cold reactants on this flow field during the first part of the cycle is evident. The injection of cold reactants is first noticed at $\tau=0.889 .^{2}$ The flow field is dominated by a large recirculation re-

\footnotetext{
2 This is caused by a higher mean pressure upstream of the combustion chamber than the mean pressure in the combustion chamber, which forces injection of reactants to occur early.
}

gion, which is particularly strong during the injection period. This recirculation region grows in strength as the injection of new reactants penetrates the combustion chamber. Note that the negative velocity associated with the recirculation zone moves downstream as the injection of new reactants penetrates the combustion chamber. The injection of new reactants begins at $\tau \simeq 0.899$ of the previous cycle and continues until $\tau \simeq 0.556$, for a total of $66 \%$ of the cycle. This is in agreement with Barr et al. [16], who showed that the injection of reactants cannot be assumed to be quasisteady, but the process continues well into the period of adverse pressure gradient.

\section{DISCUSSION}

To better show the fluid dynamic features of this flow field, particle paths were calculated from the velocity data presented in Fig. 6 . Figures $7 a$ and $7 b$ present the results from this calculation. These data were obtained by placing fluid tracers at uniformly placed spatial intervals throughout the domain and tracking them as they move under the influence of the velocity field. The particles were stepped through time using 50 time steps between each of the 18 time bins. Linear interpolation was used in time and space to determine the velocity from the measured data. The calculation was allowed to develop for a time period of $\tau=0.222$ before the desired time frame was obtained. This algorithm was repeated for each of the 18 time bins. Note that the particle paths calculated in this way do not represent a continuous path from time bin to time bin. The data shown in Figs. $7 \mathrm{a}$ and $7 \mathrm{~b}$ are multiple exposures of individual particle locations at 5 evenly spaced times representing the particle path obtained from one frame to another. For example, consider $\tau=0.222$ and the paths located at $x=50 \mathrm{~mm}, y=0 \mathrm{~mm}$. These paths represent fluid which is moving in the negative direction beginning at the right and progressing to the left. At $\tau=0.222$ the particle was at the right most position and at $\tau=0.278$ the particle had moved along the indicated path to the left most position.

The major features of this flow field are now evident. The strong injection velocity quickly 
establishes a well-defined large toroidal vortex. This toroidal vortex is best seen at $\tau=0.222$, which is the time corresponding to the maximum in the injection velocity. Also evident is the effect this toroidal vortex has on the creation of the large recirculation zone. In the early stages of development the recirculation zone is weak, but as the toroidal vortex gains strength the influence of this increased circulation causes a strong negative velocity in the center of the combustion chamber. The mechanism for the convection of reactants to the rear stagnation point of the stagnation plate is attributed to the strong circulation of this toroidal vortex at times $0.111 \leq \tau \leq 0.278$. This toroidal vortex also diverts a major portion of the flow to the walls outside of the measurement domain. This part of the flow is responsible for the convection of reactants far downstream and reentrained at large $x$ distances. The particle paths at $\tau=0.278$ and $x=60-70$ $\mathrm{mm}$ are almost radial providing a mechanism to convect fluid from the wall region into the centerline. These results show that this toroidal vortex creates a feature in the flow capable of convecting reactants to both the near and far fields where ignition of these reactants is observed to begin (Fig. 4).

As the reactant's injection rate increases, the circulation in this toroidal vortex increases. This results in a buildup of momentum in the center of the flow that overwhelms the inlet jet

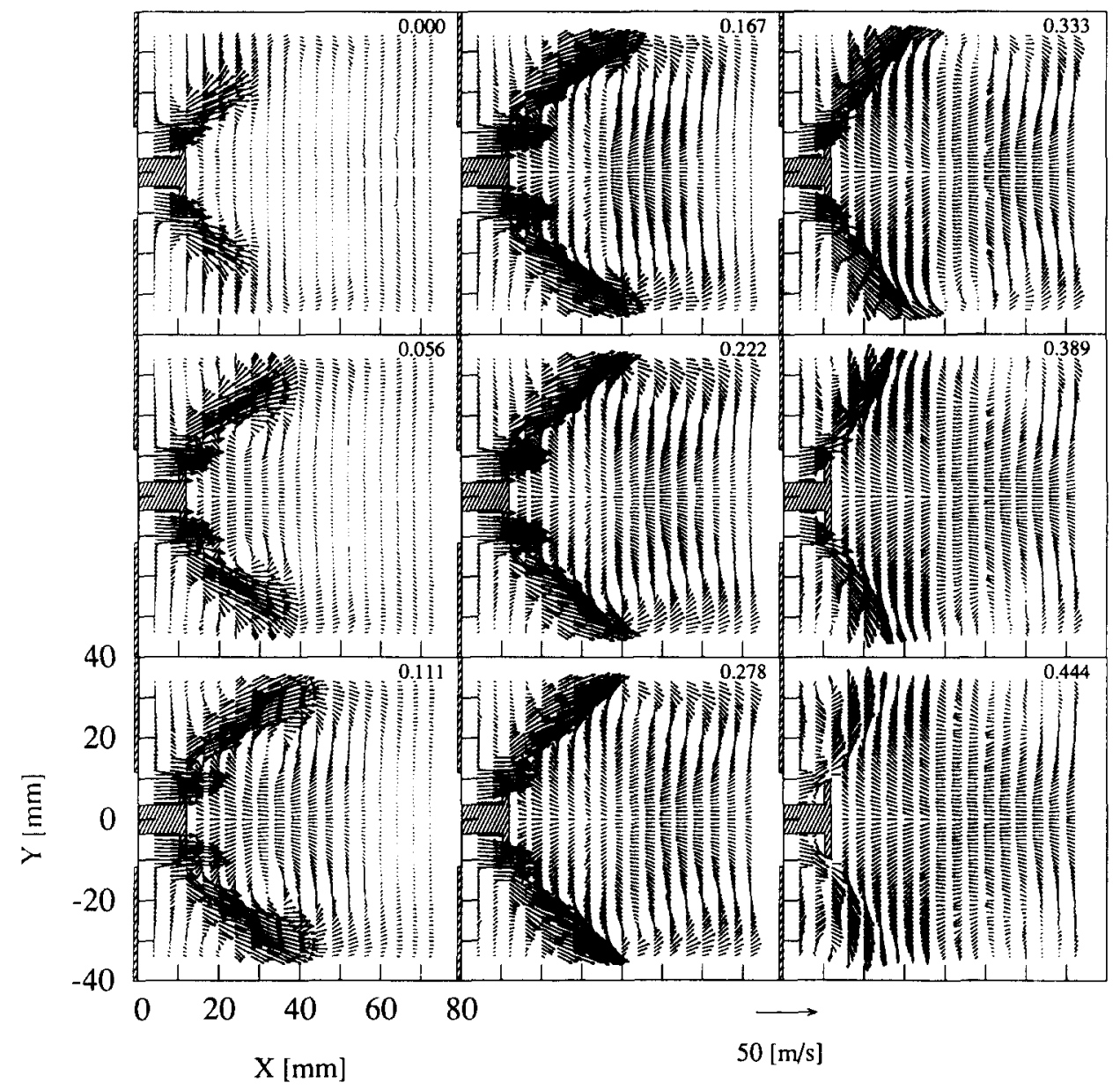

(a)

Fig. 6. Velocity field mapping in the combustion chamber for the pulsating, combusting flow. Shown are 9 individual times for the first half $(6 \mathrm{a})$ and the second half $(6 \mathrm{~b})$ of the combustor cycle. These vectors are plotted with the tails located at uniform streamwise locations. 


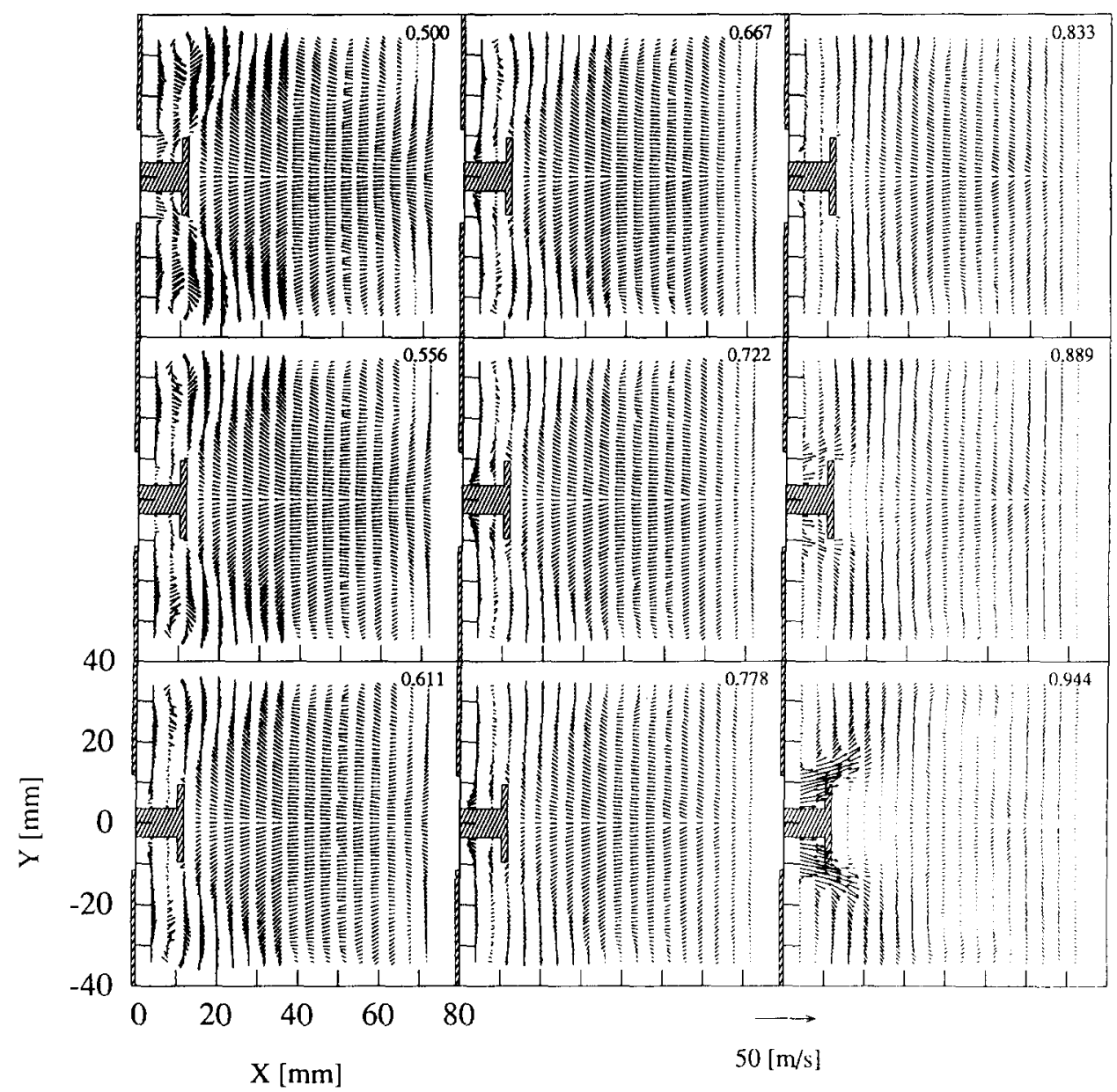

(b)

Fig. 6. (Continued)

and pushes it back toward the inlet, as indicated by the almost radial particle paths at $\tau=0.556$, and $x=20 \mathrm{~mm}$.

Another feature of this flow is a smaller toroidal vortex located in the corners near $x=0 \mathrm{~mm}$. The circulation of this smaller toroidal vortex is of the opposite sign from the toroidal vortex located in the center of the combustion chamber. This small toroidal vortex grows and decays in strength throughout the cycle, but is always present. This flow field is clearly dominated by the effects of the entrance jet.

Like the presence of the toroidal vortex, the effects of the energy release can be more readily seen in these particle paths than in the raw velocity vectors. Early in the combustion phase $(0.444 \leq \tau \leq 0.556)$ the primary toroidal vortex has grown in size and moved out toward the walls. The volumetric expansion due to combustion pushes this toroidal vortex completely out of the center of the flow and makes the flow pattern much more uniform throughout the measurement domain. (See for example the particle paths for times $\tau=0.722$ and $\tau=$ 0.778 .) During the decay of the energy release $(0.833 \leq \tau \leq 0.889)$, some residual circulation exists from the primary toroidal vortex, $x=$ 40-50 $\mathrm{mm}$.

These particle paths have been superimposed on the images of schlieren and chemiluminescence of Figs. 3 and 4 and are shown in Fig. 8 (See color plates between pages 30 and 31.). These data are shown for four key times in the cycle: the onset of injection $(\tau \simeq 0.94)$, the peak of injection $(\tau \simeq 0.22)$, the onset of 


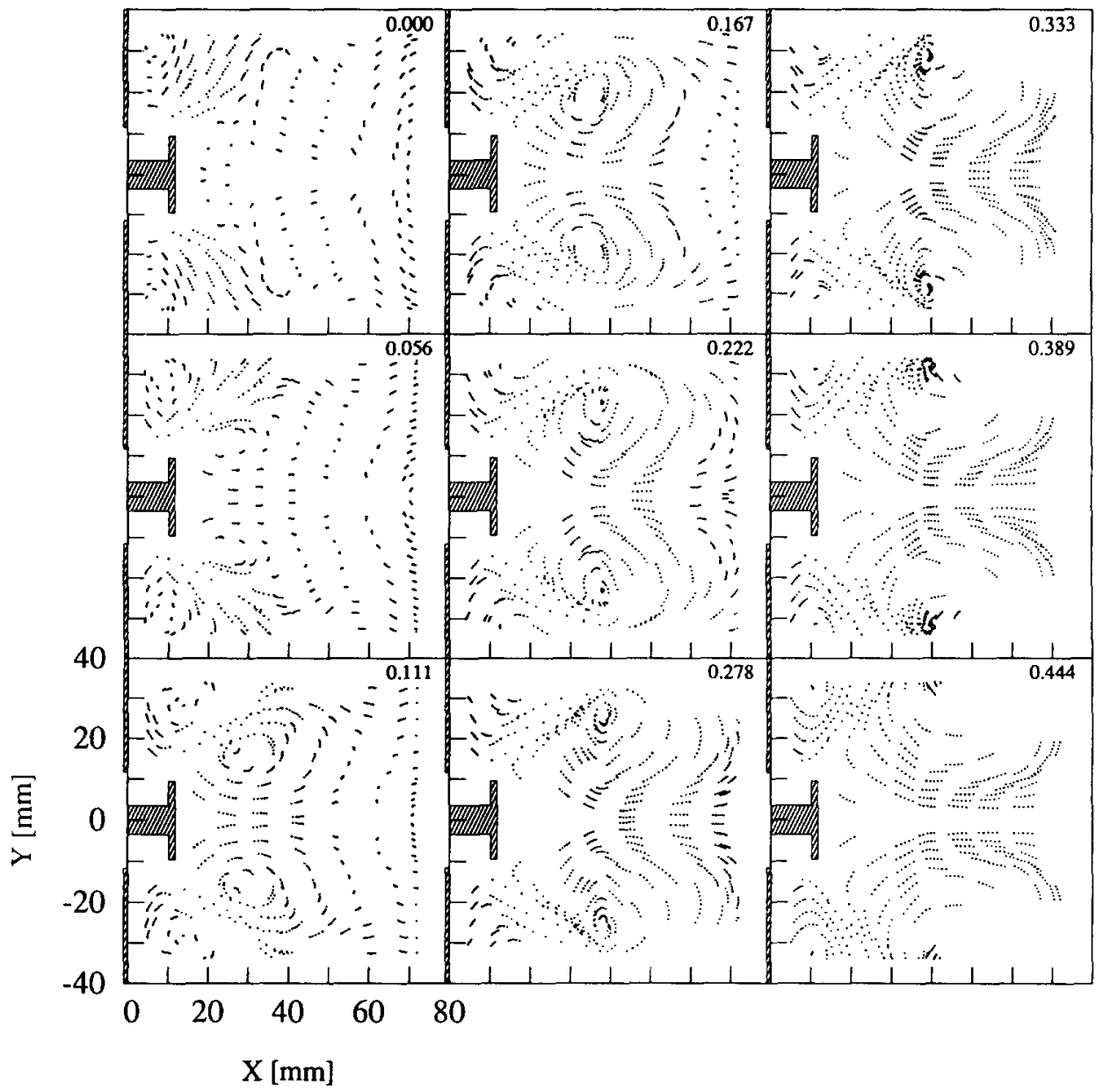

(a)

Fig. 7. Calculated particle paths in the combustion chamber for the pulsating, combusting flow. These results were obtained from the measured velocity data shown in Fig. 6. Shown are nine individual times for the first half (7a) and the second half $(7 \mathrm{~b})$ of the combustor cycle.

reaction $(\tau \simeq 0.44)$, and the peak of reaction $(\tau \simeq 0.67)$. In Fig. 8 the direction of motion for the particle paths is indicated by a color change from dark to light. Recall that these particle paths were obtained from ensemble averaged velocity data averaged over thousands of cycles, while the images represent single instantaneous shots with an exposure time of $20 \mu \mathrm{s}$. The correlation between these two data sets is remarkable.

Also shown in Fig. 8 are ensemble-averaged velocity fluctuations for both the streamwise and transverse directions. These experimental data were actually obtained with a streamwise and transverse resolution of 4 and $1 \mathrm{~mm}$, respectively. For the data shown in the Fig. 8, linear interpolation was used in the streamwise direction to obtain data on the square grid with $1 \mathrm{~mm}$ spacing. ${ }^{3}$ The peak velocity fluctuation for the streamwise direction and the transverse direction was $19 \mathrm{~m} / \mathrm{s}$ and $13 \mathrm{~m} / \mathrm{s}$, respectively; the peak of injection corresponding to an injection velocity of about $50 \mathrm{~m} / \mathrm{s}$.

The injection of the reactants and the associated velocity field is seen in the schlieren

\footnotetext{
${ }^{3}$ The dark regions shown in the Schlieren images represent cold reactants while the purple regions represent the hotter and more isothermal regions. The background in the chemiluminescence images is depicted as a gray with the lowest signal in blue changing to purple representing the maximum signal. The color scale used for the finescale velocity starts with blue representing a fluctuation of $0 \mathrm{~m} / \mathrm{s}$ progressing to yellow at a full scale of $20 \mathrm{~m} / \mathrm{s}$.
} 


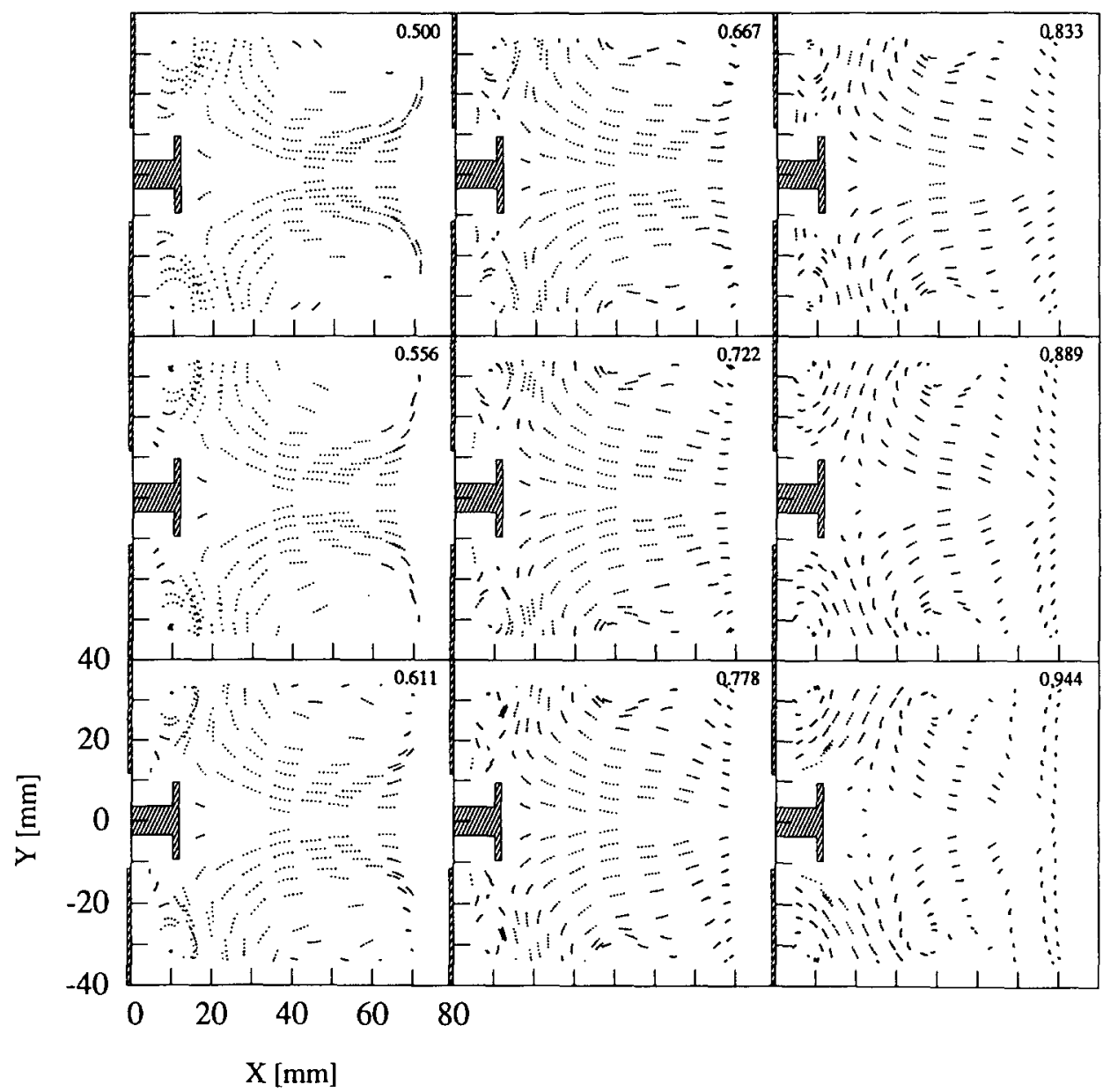

(b)

Fig. 7. (Continued)

images of Fig. 8. Shown in these images is the injection and subsequent rollup of the reactants. As the reactants mix with the residual hot products the schlieren signal is lost, suggesting that the mixture is uniformly mixed. Near the stagnation plate $(x=15 \mathrm{~mm})$ the reactants never quite mix with the residual products thoroughly enough to lose the schlieren image. However, the small toroidal vortex in the corners at $x=10 \mathrm{~mm}$ does appear to be well mixed. Careful examination of the schlieren images at the onset of injection shows that reactants near the injection port never mix with the surrounding hot fluid.

Like Fig. 4, Fig. 8 shows the spatial (integrated along the line-of-sight) and temporal location of the chemiluminescence during the combustion process. There are several enlight- ening features about these images which, when correlated with the particle paths, become clear. No reaction is observed during the period of rapid injection along the outer edges of the injection jet, even though reactants are in contact with the residual hot products. ${ }^{4}$ If the continuous combustion found near the injection jet is due to small yet discrete elements of reacting fluid, these elements would be entrained into the reactants during injection and would be a source of ignition. However, during

\footnotetext{
${ }^{4}$ Note: some of these reactants have been in contact with hot products since the beginning of the cycle or for about $2.5 \mathrm{~ms}$. This residence time is significantly longer than the chemical kinetic ignition delay time of about $1.3 \mathrm{~ms}$ for these conditions.
} 
the period of maximum injection the reaction is at a minimum ( $\tau \simeq 0.222$ in Fig. 4 , and the peak of injection in Fig. 8), showing no signs of an ignition source. Combustion of the injected reactants has been suppressed during the injection process.

The first indication of reaction $(\tau=0.387$ in Fig. 4) occurs near the downstream side of the stagnation plate, and farther downstream near the centerline of the combustion chamber on the inside of the large recirculation zone. As the reaction proceeds, the intensity rapidly increases in the stagnation region behind the plate and along the edges of the recirculation zone, until $\tau=0.611$, where it appears as though reaction is filling the combustion chamber. At $\tau=0.667$ the reaction occurs in the center of the large toroidal vortex. Ignition has occurred along the edges of this vortical structure near the centerline and at large streamwise locations, $(0.387 \leq \tau \leq 0.556)$. The initially magenta region at $\tau=0.556$ has increased in intensity to the yellow shown at $\tau=0.661$. The rest of the chemiluminescence shown in magenta at $\tau=0.611$ is assumed to be along the outer edge of this toroidal vortex and propagates into the center of the toroidal vortex core. $\tau=0.722$ shows that the combustion has consumed the reactants in the core. The reactants that were not entrained into this large vortical structure react more slowly and are reacting on the outside of this primary reaction zone, along the walls and downstream. These areas are not as strongly affected by the injection velocity, yet still have combustionstabilizing mechanisms (for example, the toroidal vortex found in the corner at $x=10$ $\mathrm{mm})^{5}$

Reaction along the injection jet's boundary has been suppressed, allowing the toroidal vortex to effectively entrain and mix the cold reactants with the residual hot products sufficiently to remove the schlieren signal from the images of Fig. 8. The appears to be reaction

\footnotetext{
${ }^{5}$ A model of continuous combustion acting as an ignition source $[10,13]$ implies that remnants of reacting elements be in the proximity of the injected reactants and entrained into the reactant stream. A conclusion that is not supported by the data presented here.
}

initiated along the outer edges of the vortical structure, then the ignition of the reactants rapidly proceeds to the center of the vortical core, and occurs in an almost volumetric fashion. This rapid movement of the combustion zone is attributed to an ignition of other reactants rather than a propagation of the reaction region seen at $\tau=0.556 .^{6}$ The maximum in tensity of reaction occurs in the vortical core, it decreases in intensity and propagates downstream with increasing cycle time to $\tau=0.833$. Subsequent reactions are noted in the small recirculation zones in the corners upstream of the stagnation plate (see Fig. 4).

A possible mechanism for the suppression of ignition along the interface between the jet and the hot products is one of stretch [24-26]. An estimate of the strain rate between the injected reactants and that of the residual products during injection is 10 times greater $\left(10,000 \mathrm{~s}^{-1}\right)$ than that required for extinction of laminar flames for the same fuel and equivalence ratio used here $\left(1000 \mathrm{~s}^{-1}\right)$ [26]. Suppressing ignition by stretch is a local phenomenon and will vary in time and space.

The existence of fine-scale velocity fluctuations during injection enhances the molecular mixing of the reactants with the residual hot products. This fine-scale motion peaks during injection, and is over by the time combustion of the reactants occurs. From the data presented here, it is seen that mixing occurs while ignition is suppressed during injection. The

\footnotetext{
${ }^{6}$ Using a flame speed of $0.7 \mathrm{~m} / \mathrm{s}$ for a premixed laminar methane air flame under these conditions [22, 23], the front would travel $350 \mu \mathrm{m}$, and yet the distances shown are on the order of $20 \mathrm{~mm}$, a factor of 57 too large. Recall from Fig. 8, that due to the high turbulent dissipation rate the fine-scale velocity fluctuations are dissipated to near 0 by the time combustion occurs. Even so, if a turbulent flame speed of $7 \mathrm{~m} / \mathrm{s}$ were used the distance traveled by this flame front would be $3.5 \mathrm{~mm}$, which is still a factor of 5.7 too short.

${ }^{7}$ The assumption that flame extinction by stretch is a dominant mechanism that contributes to an appropriate ignition delay is discussed in greater detail in the companion paper by Barr and Keller [27]. However, based on the experimental evidence presented so far, one can only assume that extinction due to stretch is one of the mechanisms that contributes to the required ignition delay.
} 
mechanism to suppress the ignition of fresh reactants is assumed to be extinction by flame stretch caused by the high strain rates. By assuming unity for the turbulent Schmidt number and knowing that the fine-scale fluctuations have dissipated by the end of the injection period, the reactants can be assumed to be well mixed by the end of the injection period. This indicates the velocity oscillations affect the system before combustion occurs and not during the combustion portion of the cycle.

The evidence presented here suggests that the ignition process in this resonant flow field is really comprised of two primary steps. The onset of ignition is suppressed during the injection process (the mechanism proposed here is one of fluid dynamic stretch) and the reactants and hot products are mixed on a fine scale heating up the reactants to an ignition temperature. As the combustion chamber pressure increases the injection velocity decreases, decreasing the strain rate, and allowing ignition of the mixed reactants and products to be correctly phased to reinforce the pressure oscillations. The reactants appear to ignite by thermal ignition in an almost volumetric fashion. These data support the concept of combustion occurring in an ensemble of well-stirred reactors, undergoing temporally staged ignition, rather than a rapidly propagating flame sheet.

This proposed delay mechanism is similar to the extinction and/or reignition process in the combustion torch experiment of Cattolica and Vosen [28]. They examined several different torch Reynolds numbers from laminar to turbulent conditions. In the lowest Reynolds number case extinction of the reaction was found in regions of high stretch, located at the stagnation point of the impulsively started jet. Reactants were convected away from the excessive strain rate region into the low strain rate vortex core, where significant reaction occurred. In the highest Reynolds number case, the impulsively started jet rapidly distributed products into a volume of premixed reactants. However, combustion was suppressed during this rapid injection of products, again the suggested mechanism was one of stretch. They found that as the injection velocity decreased, thereby decreasing the strain rate, ignition of the reactant and product mixture occurred.
Note, a residual radical pool could play a significant roll in this stretch/reignition process. However, no data exist to indicate how important this radical pool might be. Therefore, for this work the thermal ignition process described above could be one in which a radical pool plays a significant roll.

Recall that both the schlieren and the chemiluminescence data are line-of-sight measurements that will mask the existence of flamelet-like structures, should they exist. One can imagine an ensemble of well-stirred reactors each undergoing temporally staged ignition, where a flamelet-like structure traverses the small well-stirred reactor. One can also imagine that ignition occurs in this well-stirred reactor in a spatially homogeneous manner or as a distributed reaction. From the schlieren and chemiluminescence data presented here no determination can be made between these two combustion modes.

The fluid dynamic flow field in the combustion chamber for an isolated cycle is a direct result of the resonant pressure field. The primary elements of this flow field are created early in the cycle during the injection of the reactants as a direct result of the favorable pressure gradient between the combustion chamber and the inlet. It is the injection of these reactants that establishes the toroidal vortex and the resultant flow field. As a result of the flow in acoustic resonance, the combustion chamber pressure begins to rise before ignition of the reactants occurs. Indeed it is this rising pressure that creates the adverse pressure gradient during the second half of the cycle. This adverse pressure gradient decelerates the inlet jet and eventually stops the injection process decreasing the strain rate, thus allowing the ignition of the reactants. This fluid dynamic behavior is a direct result of the oscillating combustion chamber pressure and the resulting pressure gradient between the inlet and the combustion chamber, and hence, it exists whether or not a combustion event occurs during the current cycle. Considering only an isolated cycle the rising pressure in the combustion chamber is a result of the flow in acoustic resonance and not the combustion process. However, this energy release, if properly phased, does reinforce the resonant pres- 
sure field in preparation for the next cycle. The resonant pressure field creates a fluid dynamic flow field that controls combustion so that the energy released is in phase with the resonant pressure field to satisfy Rayleigh's criterion, the requirement necessary to maintain the stable limit cycle.

The previous discussion is not to imply that the fluid dynamics and the combustion processes are decoupled, this clearly is not the case. However, the coupling does occur as a result of Rayleigh's criterion over multiple cycles. The events that occur during the current cycle are a result of the resonant pressure field that was reinforced by the combustion event from the previous cycle. If the pressure field was not previously reinforced adequately then the current fluid dynamic flow field would be modified, affecting the current combustion event which in turns modifies the pressure signature for the succeeding cycle. For improperly phased events the volumetric expansion due to combustion will cause the pressure signature to differ from its typical sinusoidal shape, resulting in a modification of the operating frequency $[6,7]$. However, this occurs late in the cycle after the favorable pressure gradient part of the cycle during which time the dominate features of the flow field were established. The events for the current cycle are a result of the resonant pressure field driven by the combustion process from previous cycles. The primary role for the combustion process is to reinforce the resonant pressure field, to overcome the associated acoustic losses. This conclusion is further supported by the analysis presented in the companion paper by Barr and Keller [27].

\section{SUMMARY}

An experimental investigation into the detailed mixing, combustion and ignition processes occurring in a flow field under the influence of resonant acoustic perturbations has been performed. These acoustic perturbations were created by a "Helmholtz" type pulse combustor. Detailed cycle-resolved measurements of the velocity field, combined with cycle-resolved single-shot images of both chemiluminescence and schlieren photographs, were presented. The ve- locity data were used to calculate particle paths, which were combined with the image data to provide a clear picture of the combustion fluid dynamics occurring inside the pulse combustor's combustion chamber.

During the first two thirds of the cycle the inlet jet forms a well-defined large toroidal vortex that is phase-locked with the combustor cycle. This vortical structure is responsible for the convection and mixing of the reactants with the residual hot products. Although the chemiluminescence is always present in quiescent regions of combustor, no luminosity exists in the region of the reactants during injection. Moreover, ignition of the fresh reactants occurs first along the outer edges of the rolled-up toroidal vortex, and not in the small corner recirculation regions of the flow. The combustion of these reactants develops rapidly in the center of this rolled up toroidal vortex where uniform combustion of the fresh reactants occurs.

This flow field is dominated by a strong toroidal vortex which entrains, on a large scale, and mixes, on a fine scale, the fresh reactants with the hot products, preparing them for a rapid, almost volumetric, combustion process initiated by thermal and/or radical ignition.

Local extinction by flame stretch is proposed as a mechanism to delay the onset of ignition during injection and to allow time for the reactants to mix with the hot products. After the injection of reactants, the strain rate decreases, allowing the reactants to undergo thermal ignition in an almost volumetric fashion. Reaction was found to exist in the smaller recirculation region behind the sudden expansion, outside the high-strain region, producing a continuous combustion source, but not the source of reignition.

We wish to thank T. T. Bramlette for his enthusiastic technical support during the development of this work. This work was performed at the Combustion Research Facility Sandia National Laboratories and supported by the U.S. Department of Energy, Office of Industrial Technologies, Advanced Industrial Concepts Division. R. S. Gemmen's support was provided by the Gas Research Institute, Department of Physical Sciences. 


\section{REFERENCES}

1. Keller, J. O., Vaneveld, L., Korschelt, D., Hubbard, G. L., Ghonem, A. F., Daily, J, W., and Oppenheim, A. K., ALAA J. 20:254 (1982).

2. Poinsot, T., Veynante, D., Bourienne, F., Candel, S., Esposito, E., and Surget, J., Twenty-Second Symposium (International) on Combustion, The Combustion Institute, Pittsburgh, 1988, pp. 1363-1370.

3. Bhafouriana, A., Frost, J., and Daily, J. W., The Joint Spring Technical Meeting of the Western States/ Canadian Sections of the Combustion Institute, Baniff, Alberta Canada, 1990.

4. Smith, O. L., Marchant, R., Willis, J., Lee, L. M., Logan, P., and Karagozian, A. R., Combust. Sci. Technol. 74:199 (1990).

5. Logan, P., Lee, J. W., Lee, L. M., Karagozian, A. R., and Smith, O. L., Combust. Flame 84:93 (1991).

6. Keller, J. O., Bramlette, T. T., Dec, J. E., and Westbrook, C. K., Combust. Flame 75:33-44 (1989).

7. Keller, J. O., Bramlette, T. T., Dec, J. E., and Westbrook, C. K., Combust. Flame 79:151-161 (1990).

8. Dec, J. E., Keller, J. O., and Hongo, I., Combust. Flame 83:271-292 (1991).

9. Oran, E. S., and Gardner, J. H., Prog. Energ. Combust. Sci. 11:253-276 (1985).

10. Putnam, A. A., Belles F. E., and Kentfield, J. A. C., Prog. Energ. Combust. Sci. 12:43-79 (1986).

11. Keller, J. O., Saito, K., and Kishimoto, K., 1984 Fall Meeting of the Western States Section of the Combustion Institute, Stanford, CA.

12. Keller, J. O., and Saito, K., Combust. Sci. Technol. 53:137-163 (1987).

13. Reuter, D., Daniel, B. R., Jagoda, J., and Zinn, B. T., Combust. Flame 65:281-290 (1986).

14. Belles, F. E., The Gas Research Institute, March 15, 1990, GRI-89/0106.

15. Ohiwa, N., Yamaguchi, S., Hasegawa, T., Kui, Q. B., and Okada, A., Jpn. Soc. Mech. Eng. Series B, No. 85-0524, 1913-1922 (1985).

16. Barr, P. K., Keller, J. O., Bramlette, T. T., Westbrook, C. K., and Dec, J. E., Combust. Flame 82:252-269 (1990).

17. Keller, J. O., and Hongo, I., Combust. Flame 80:219-237 (1990).

18. Dec. J. E., and Keller, J. O., Combust. Flame 80:358-371 (1990).

19. Keller, J. O., and Westbrook, C. K., Twenty-First Symposium (International) on Combustion, The Combustion Institute, Pittsburgh, 1987, pp. 547-555.

20. Gaydon, A. G., The Spectroscopy of Flames, Chapman and Hall, 1974.

21. Child, E. T., and Wohl, K., Seventh Symposium (International) on Combustion, The Combustion Institute, Pittsburgh, 1959, pp. 215-220.

22. Warnatz, J., Eighteen Symposium (International) on Combustion, The Combustion Institute, Pittsburgh, 1981, p. 369.

23. Kanury, A., Murty, Introduction to Combustion Phenomena, Gordon \& Breach, New York, 1975, p. 290.

24. Peters N., Twenty-First Symposium (International) on Combustion, The Combustion Institute, Pittsburgh, 1987, pp. 1231-1250.

25. Law, C. K., Zhu, D. L., and Yu, G., Twenty-First Symposium (Intemational) on Combustion, The Combustion Institute, Pittsburgh, 1987, pp. 1419-1426.

26. Kee, R. J., Miller, J. A., and Evans, G. H., TwentySecond Symposium (International) on Combustion, The Combustion Institute, Pittsburgh, 1988, pp. 1479-1494.

27. Barr, P. K., and Keller, J. O., Combust. Flame 99:43-52 (1994).

28. Cattolica, R., Vosen, S., Combust. Flame 68:267-286 (1988).

Received 1 April 1991; revised 1 March 1993 